\title{
Optical observations of enhanced activity of the 2005 Draconid meteor shower
}

\author{
P. Koten, J. Borovička, P. Spurný, and R. Štork \\ Astronomical Institute of the Academy of Sciences, Ondřejov Observatory, Fričova 298, 25165 Ondřejov, Czech Republic \\ e-mail: koten@asu.cas.cz
}

Received 29 November 2006 / Accepted 11 January 2007

\begin{abstract}
Context. The enhanced activity of the Draconid meteor shower was observed on October 8, 2005 using video and photographic cameras.

Aims. The aim of this paper is to use a higher than usual number of recorded meteors to look at some physical properties of the Draconid meteoroids, to describe the activity profile, and to infer meteor orbits.

Methods. Video data on meteors are used for the determination of the meteor shower activity. Double station data provide precise beginning heights of the meteors as well as their radiants and orbits. Beginning heights and light curves of all meteors are used for investigation of meteoroid properties.

Results. Only the descending branch of the enhanced activity was observed between 17:30 and 19 UT. The mass distribution index is similar to the 1998 return. Beginning heights of the Draconid meteors are several kilometres higher in comparison with other meteors of similar velocity. Light curves are nearly symmetrical, with a slight preference of early maxima. Both results are consistent with the very fragile nature of Draconid meteoroids.
\end{abstract}

Key words. meteors, meteoroids - comets: individual: 21P/Giacobini-Zinner

\section{Introduction}

The Draconid or Giacobinid meteor shower is a minor periodic meteor shower, which sometimes produces brief but spectacular meteor storms. Such storms occurred twice during last century, in 1933 and 1946 (e.g., Jacchia et al. 1950). Lower activity was observed in 1952 (Davies \& Lovell 1955), whereas in 1985 and 1998 the shower produced moderate meteor rates (Arlt 1998). The shower is active between October 6th and 10th, with a maximum occurring between October 8 th and 9 th. The activity of the meteor shower depends on the position of the parent comet 21P/Giacobini-Zinner on its orbit.

In 2005 the parent comet returned to perihelion in July, but it was not clear whether it would influence activity of the Draconids that year. According to the modeling of the Draconids (http://www.imcee.fr/, J. Vaubaillon), no storm was expected to happen in October 2005. Nevertheless an enhancement due to the proximity of the comet to perihelion was not possible to exclude. Thus the observers were encouraged to look for some signs of higher activity of this shower.

Campbell-Brown et al. (2006) reported the Draconid outburst, which occurred around the solar longitude $\lambda_{\odot}=195.42^{\circ}$ corresponding to 16.1 UT, October 8, 2005. Between 14.5 UT and at least $18 \mathrm{UT}$, the CMOR radar detected meteor activity significantly above the sporadic background at multiple frequencies. The equivalent hourly binned zenithal hourly rate (ZHR) during the peak of the activity was higher than 150 . The enhancement, although not as strong as that found in the radar data, was also observed by visual observers located in Asia, Europe, and North America. The visual observations provided ZHR of 40. Refined numerical simulation of the Draconid shower, which additionally included smaller particles, identified that the 2005 outburst was caused by the material released during the 1946 perihelion passage of the parent comet.

In this paper we report the results of the double-station video experiment, which was carried out in the Czech Republic during the time of the Draconid meteor shower activity. Despite variable weather conditions, the enhanced activity was detected and simultaneous data on some of the Draconid meteors recorded. Due to later sunset in Central Europe only the descending branch of the activity curve was probably observed. Clouds did not allow us to precisely determine if the peak still occurred before the observation started or was already observed.

We discuss here the activity profile, mass distribution, radiants and orbits, beginning heights, and light curves of meteors. The second paper (Borovička et al. 2007) is devoted to detailed study of atmospheric deceleration we detected for the meteors and also to Draconid spectra.

\section{Instrumentation, observation, data processing}

The double-station video observations were carried out on the base Ondřejov - Třebíc during three consecutive nights between October 6th and 9th, 2005. Both stations are separated by the distance of $109.3 \mathrm{~km}$, and the azimuth of the second station is $314.3^{\circ}$ (azimuth of south $=0^{\circ}$ ). Each station was equipped with two videocameras, which were connected to the 2 nd generation image intensifiers. At the Ondřejov observatory, the direct camera equipped with a Mullard XX1332 intensifier and a Jupiter $2 / 85 \mathrm{~mm}$ lens provided a field-of-view of $32^{\circ}$. The second camera, connected to the Dedal-41 intensifier with an Arsat $1.4 / 50 \mathrm{~mm}$ lens and spectral grating with 600 grooves $/ \mathrm{mm}$ 
Table 1. Atmospheric trajectories and physical properties of Draconid video meteors. $\alpha_{\mathrm{G}}$ and $\delta_{\mathrm{G}}$ are coordinates of the geocentric radiant, $v_{\mathrm{G}}$ geocentric velocity, $M_{\max }$ absolute maximum brightness, $m_{\text {phot }}$ photometric mass, $H_{\mathrm{B}}$ beginning height, $K_{\mathrm{B}}$ parameter according to Ceplecha (1988), $H_{\mathrm{E}}$ terminal height, $\cos (z)$ cosine of the zenith distance of the radiant, and $F$ parameter describing the meteor light curve shape. All meteors were observed in the night of October 8/9, 2005.

\begin{tabular}{|c|c|c|c|c|c|c|c|c|c|c|c|}
\hline Meteor & $\begin{array}{l}\text { Time } \\
\text { [UT] }\end{array}$ & $\begin{array}{l}\alpha_{\mathrm{G}} \\
{\left[{ }^{\circ}\right]}\end{array}$ & $\begin{array}{l}\delta_{\mathrm{G}} \\
{\left[{ }^{\circ}\right]}\end{array}$ & $\begin{array}{l}\left(v_{\mathrm{G}}\right)^{*} \\
{\left[\mathrm{~km} \mathrm{~s}^{-1}\right]}\end{array}$ & $\begin{array}{l}M_{\max } \\
{[\mathrm{mag}]}\end{array}$ & $\begin{array}{l}m_{\text {phot }} \\
{[\mathrm{g}]}\end{array}$ & $\begin{array}{l}H_{\mathrm{B}} \\
{[\mathrm{km}]}\end{array}$ & $\overline{K_{\mathrm{B}}}$ & $\begin{array}{l}H_{\mathrm{E}} \\
{[\mathrm{km}]}\end{array}$ & $\overline{c \cos (z)}$ & $\overline{F F}$ \\
\hline 05A08030 & $18: 55: 02$ & $264.0 \pm 1.0$ & $55.2 \pm 0.6$ & $20.9 \pm 0.6$ & 3.6 & 0.006 & 100.5 & 6.76 & 92.0 & 0.87 & 0.40 \\
\hline 05A08054 & $20: 33: 13$ & $263.2 \pm 0.3$ & $55.26 \pm 0.08$ & $21.0 \pm 0.2$ & 1.9 & 0.03 & 101.8 & 6.70 & 88.7 & 0.74 & 0.52 \\
\hline 05A08057 & 20:39:31 & $264.5 \pm 0.3$ & $55.2 \pm 0.2$ & $21.0 \pm 0.2$ & 2.0 & 0.05 & 105.2 & 6.47 & 87.3 & 0.74 & 0.46 \\
\hline 05A08078 & 21:59:01 & $261.1 \pm 0.5$ & $55.67 \pm 0.09$ & $21.0 \pm 0.3$ & 2.5 & 0.04 & 102.6 & 6.70 & 88.6 & 0.61 & 0.64 \\
\hline 05A08096 & $22: 56: 44$ & $264.3 \pm 2.8$ & $55.8 \pm 0.7$ & $21.0 \pm 0.5$ & 3.7 & 0.012 & 102.0 & 6.76 & 92.9 & 0.55 & 0.24 \\
\hline 05A08128 & $00: 13: 22$ & $262.7 \pm 0.2$ & $55.28 \pm 0.16$ & $20.9 \pm 0.5$ & 3.5 & 0.016 & 104.0 & 6.66 & 91.0 & 0.44 & 0.31 \\
\hline
\end{tabular}

* Computed on the assumption of $v_{\infty}=23.57 \mathrm{~km} \mathrm{~s}^{-1}$.

Table 2. Heliocentric orbits (J2000.0) of the Draconid video meteors. $D_{\mathrm{SH}}$ is the orbital similarity criterion computed according to Southworth \& Hawkins (1967). The orbit of the comet 21P/Giacobini-Zinner (2005) provided by the JPL Horizons program was used as the reference orbit.

\begin{tabular}{llllllll}
\hline \hline Meteor & $a[\mathrm{AU}]$ & $e$ & $q[\mathrm{AU}]$ & $\omega\left[^{\circ}\right]$ & $\Omega\left[^{\circ}\right]$ & $i\left[^{\circ}\right]$ & $D_{\mathrm{SH}}$ \\
\hline 05A08030 & $3.7 \pm 0.5$ & $0.73 \pm 0.04$ & $0.9965 \pm 0.0006$ & $173.8 \pm 0.7$ & 195.517 & $31.5 \pm 0.7$ & 0.05 \\
05A08054 & $3.7 \pm 0.2$ & $0.73 \pm 0.013$ & $0.9960 \pm 0.0002$ & $173.3 \pm 0.2$ & 195.585 & $31.6 \pm 0.3$ & 0.05 \\
05A08057 & $3.8 \pm 0.2$ & $0.739 \pm 0.014$ & $0.9968 \pm 0.0001$ & $174.2 \pm 0.2$ & 195.589 & $31.6 \pm 0.3$ & 0.04 \\
05A08078 & $3.4 \pm 0.2$ & $0.71 \pm 0.02$ & $0.9948 \pm 0.0003$ & $171.9 \pm 0.3$ & 195.643 & $31.9 \pm 0.4$ & 0.04 \\
05A08096 & $3.6 \pm 0.4$ & $0.72 \pm 0.03$ & $0.9968 \pm 0.0015$ & $174 \pm 2$ & 195.683 & $31.8 \pm 0.6$ & 0.03 \\
05A08128 & $3.5 \pm 0.3$ & $0.72 \pm 0.02$ & $0.9957 \pm 0.0001$ & $172.9 \pm 0.2$ & 195.736 & $31.5 \pm 0.5$ & 0.05 \\
\hline Comet & 3.526 & 0.706 & 1.038 & 172.535 & 195.423 & 31.811 & - \\
\hline
\end{tabular}

was used for meteor spectroscopy. Both cameras on the second station were coupled to Dedal-41 intensifiers. The first one, equipped with an Arsat 1.4/50 mm lens, provided a field-of-view of $28^{\circ}$ in diameter. The second one connected with a Zenitar 2.8/16 mm lens served as a wide-field system with a field-ofview of about $85^{\circ}$. In addition to the video observations, one bright Draconid meteor was also recorded by three all-sky photographic cameras of the Czech part of the European fireball network (see Spurný et al. 2007 for network description).

The recorded video data were stored on S-VHS video tapes. These tapes were searched using automatic meteor detection software MetRec (Molau 1999). Meteor records were digitalized with resolution 25 images per second and $768 \times 576$ pixels. Semi-automatic measurement software MetPho (Koten 2002) was used for the measurement of the records. The output provides not only positional measurements, but also photometry of the meteor. Atmospheric trajectories and heliocentric orbits were first computed using our standard procedures for video meteors. At this moment we recognized that significant meteor deceleration strongly influences the results. We investigated this problem more deeply. Another paper, which analyses this phenomenon and its implication for the meteoroid structure is in preparation (Borovička et al. 2007). To compute heliocentric orbits in this paper we took the initial velocity of the photographic fireball, which does not show deceleration and has an orbit that is determined very precisely, as the initial velocity for all video meteors. The video data proved to be consistent with this assumption (Borovička et al. 2007).

The meteor shower membership was determined using the Southworth-Hawkins $D_{\mathrm{SH}}$ criterion (Southworth \& Hawkins 1967). The orbit of the parent comet taken from JPL Horizons web program (http://ssd.jpl.nasa.gov/horizons.cgi) was used as the reference orbit. All meteors satisfying condition $D_{\mathrm{SH}}<0.20$ were considered as Draconids. The light curves were used for determination of the photometric mass using luminous efficiency according to Ceplecha (1988).

\section{Recorded data}

The detection software MetRec has relatively large tolerance in the shower assignment and identified 12 video meteors observed from both stations as possible Draconid shower members. More precise measurement and reduction of these records confirmed 7 of them as real Draconids. One of them was recorded only in the zero order of the spectral camera and by the wide-field camera. Unfortunately, the small angle of planes resulted in a big trajectory uncertainty and this meteor was finally excluded from the sample. Atmospheric trajectories and physical properties of the six remaining double station Draconid meteors are summarized in Table 1. Table 2 shows heliocentric orbits of these meteors, and Table 4 provides geophysical and heliocentric data on the photographically recorded meteor.

26 single station meteors were labeled by MetRec as the Draconid members. They were measured, too, and it was decided, according to their angular velocity and to the distance of the prolonged path from the radiant, whether they could belong to the Draconid meteor shower. 10 of them were rejected; 16 were supposed to be suitable for another analysis. Using mean values of apparent radiants and velocities of the double station meteors the atmospheric trajectories of the single station ones were calculated. Results are given in Table 3. We should note that the values of the beginning height of the single station meteors are only approximate and in comparison with the same quantity of the double station meteors, which are typically determined with the precision of $0.1 \mathrm{~km}$, these are affected by much bigger errors. The estimate is that the single station data may differ from real values up to $5 \mathrm{~km}$. Subsequently the value of the parameter $K_{\mathrm{B}}$ (Sect. 4.4) is affected in the same way. Nevertheless the comparison with more precise double station data shows that these approximated values are reasonable and we can use them for some kind of analyses. The photometric mass is not affected by this uncertainty neither is the parameter $F$ (Sect. 4.5). 
Table 3. Data on the single station meteors recorded by the Ondřejov direct camera.

\begin{tabular}{|c|c|c|c|c|c|c|c|c|}
\hline Meteor & $\begin{array}{l}\text { Date } \\
2005\end{array}$ & $\begin{array}{l}\text { Time } \\
\text { [UT] }\end{array}$ & $\begin{array}{l}m_{\text {phot }} \\
{[\mathrm{g}]}\end{array}$ & $\begin{array}{l}M_{\max } \\
{[\mathrm{mag}]}\end{array}$ & $\begin{array}{l}H_{\mathrm{B}} \\
{[\mathrm{km}]}\end{array}$ & $\overline{K_{\mathrm{B}}}$ & $\begin{array}{l}H_{\mathrm{E}} \\
{[\mathrm{km}]}\end{array}$ & $F$ \\
\hline $05 \mathrm{~A} 06067$ & 6.10. & $22: 12: 59$ & 0.013 & 3.3 & 101 & 6.8 & 90 & 0.51 \\
\hline 05A06161 & 7.10 & 01:07:59 & 0.0055 & 4.0 & 109 & 6.2 & 106 & 0.47 \\
\hline 05A07073 & 7.10 . & 21:54:03 & 0.007 & 3.8 & 100 & 6.9 & 93 & 0.54 \\
\hline 05A07088 & 7.10 . & $22: 27: 07$ & 0.0073 & 4.0 & 107 & 6.3 & 98 & 0.52 \\
\hline 05A07120 & 7.10 . & 23:37:09 & 0.0076 & 4.2 & 109 & 6.0 & 106 & 0.39 \\
\hline 05A07129 & 7.10 . & $23: 52: 53$ & 0.0027 & 4.7 & 105 & 6.5 & 102 & - \\
\hline 05A08001 & 8.10 . & $17: 36: 50$ & 0.0031 & 4.6 & 102 & 6.6 & 93 & - \\
\hline 05A08002 & 8.10 . & $17: 40: 52$ & 0.0016 & 3.8 & 103 & 6.5 & 89 & 0.35 \\
\hline 05A08007 & 8.10 . & $17: 51: 02$ & 0.013 & 2.8 & 106 & 6.8 & 98 & 0.27 \\
\hline 05A08008 & 8.10 . & $17: 59: 00$ & 0.028 & 2.4 & 108 & 6.2 & 96 & 0.43 \\
\hline $05 \mathrm{~A} 08013$ & 8.10 . & $18: 24: 52$ & 0.016 & 2.8 & 98 & 7.0 & 85 & 0.70 \\
\hline 05A08014 & 8.10. & 18:32:16 & 0.0027 & 4.4 & 107 & 6.3 & 98 & - \\
\hline 05A08015 & 8.10 . & $18: 32: 37$ & 0.037 & 2.6 & 105 & 6.4 & 84 & 0.54 \\
\hline 05A08017 & 8.10 . & $18: 34: 54$ & 0.0008 & 5.4 & 101 & 6.7 & 96 & - \\
\hline 05A08020 & 8.10 . & 18:39:04 & 0.0033 & 4.4 & 107 & 6.2 & 98 & - \\
\hline 05A08024 & 8.10 . & $18: 45: 30$ & 0.004 & 4.1 & 97 & 7.0 & 89 & 0.55 \\
\hline
\end{tabular}

Table 4. Atmospheric trajectory, geocentric radiant, and heliocentric orbit (J2000.0) of the photographic Draconid meteor. The notation has the same meaning as in Tables 1 and 2. For the classification of the fireballs, the $P_{\mathrm{E}}$ criterion is usually used (Ceplecha 1988). According to this criterion the fireball EN081005B belongs to the group IIIB.

\begin{tabular}{ll}
\hline \hline Meteor & EN081005B \\
Date & October 8,2005 \\
Time $[\mathrm{UT}]$ & $18: 46: 04.2 \pm 0.1^{*}$ \\
$\alpha_{\mathrm{G}}\left[^{\circ}\right]$ & $262.96 \pm 0.17$ \\
$\delta_{\mathrm{G}}\left[{ }^{\circ}\right]$ & $55.67 \pm 0.07$ \\
$v_{\mathrm{G}}[\mathrm{km} / \mathrm{s}]$ & $20.93 \pm 0.09$ \\
$M_{\mathrm{Max}}[\mathrm{mag}]$ & -5.5 \\
$m_{\infty}[\mathrm{g}]$ & 10 \\
$H_{\mathrm{B}}[\mathrm{km}]$ & 98.37 \\
$H_{\mathrm{E}}[\mathrm{km}]$ & 83.11 \\
$\mathrm{PE} \mathrm{criterion}$ & -6.38 \\
\hline$a[\mathrm{AU}]$ & $3.53 \pm 0.07$ \\
$e$ & $0.717 \pm 0.005$ \\
$q[\mathrm{AU}]$ & $0.99606 \pm 0.00010$ \\
$\omega\left[{ }^{\circ}\right]$ & $173.25 \pm 0.12$ \\
$\Omega\left[^{\circ}\right]$ & $195.51097 \pm 0.00001$ \\
$i\left[^{\circ}\right]$ & $31.74 \pm 0.10$ \\
$D_{\mathrm{SH}}$ & 0.02 \\
\hline
\end{tabular}

* The beginning of the meteor.

\section{Analyses of data}

\subsection{Shower activity}

Tables 1 and 3 show that a large number of reported Draconid meteors occurred on October 8 around 18 UT. The Draconid annual meteor shower usually does not show activity with ZHR $>1$ (Jenniskens 1995). The enhancement of the activity is even more noticeable in comparison with previous two nights, when only several randomly separated Draconids were observed (Fig. 1).

Due to later sunset in our country the observation started at 17:32 UT. Unfortunately it was partly cloudy at the Ondřejov station and almost overcast at the Třebíč station at this time. Cloudiness changed quickly during the first half of the night and influenced observed numbers of the meteors. Despite not ideal weather conditions, we tried to construct the activity profile of the meteor shower. Only meteors recorded by the Ondřejov direct camera were used for this attempt. They were binned into half hour intervals. The rough numbers were corrected to the zenith distance of the radiant, which significantly changed during the night. For each time interval the cloudiness was also evaluated and its influence on the total numbers was estimated. We keep in mind that the correction to the cloudiness is only approximate, which is nicely illustrated by the artificial "peak" around 23 UT. This peak was caused by only one meteor, which appeared in the hole between the clouds at the time when the overall cloudiness in the field-of-view was very high.

Figure 1 shows that only the end of the enhanced activity was very probably observed. According to this plot the enhancement of the activity ended at about 19 UT. The cloudiness after 19 UT was low and the drop in activity is real. For the rest of the night the numbers of meteors did not exceed the annual Draconid level. This result is in good agreement with the observations of Campbell-Brown et al. (2006). According to their paper the maximum activity occurred at 16.1 UT. Their activity curve ceased after 18 UT when the sensitivity of the radar dramatically decreased. Nevertheless, the activity was still significantly above the background at that moment. This is consistent with our data showing that the activity continued for at least one more hour.

\subsection{Masses}

Tables 1 and 3 show that the recorded meteors cover a range of photometric masses between $8 \times 10^{-4}$ and $4.9 \times 10^{-2}$ grams. Although the total number of the Draconid meteors is rather small, we tried to determine the mass distribution index of the meteor shower. For this purpose we used the generally accepted form of the mass distribution

$\mathrm{d} N=C m^{-s} \mathrm{~d} m$

where $\mathrm{d} N$ is the number of meteors with masses between $m$ and $m+\mathrm{d} m$ and $C$ is a constant. We used the integrated form of this equation and determined the slope of the plot of the logarithm of the cumulative number of meteors as a function of the logarithm of the photometric mass. If the slope is $k$ then the mass distribution index $s=1-k$.

The mass distribution is plotted in Fig. 2. As usual, there is a bias against detection of the faintest meteors in the sample, which causes the distribution not to be linear within whole range of the masses. Therefore the linear fit is applied only on the meteors with a logarithm of the photometric mass above -2.3 . The slope of this fit is $k=-0.78$, which results in the mass 

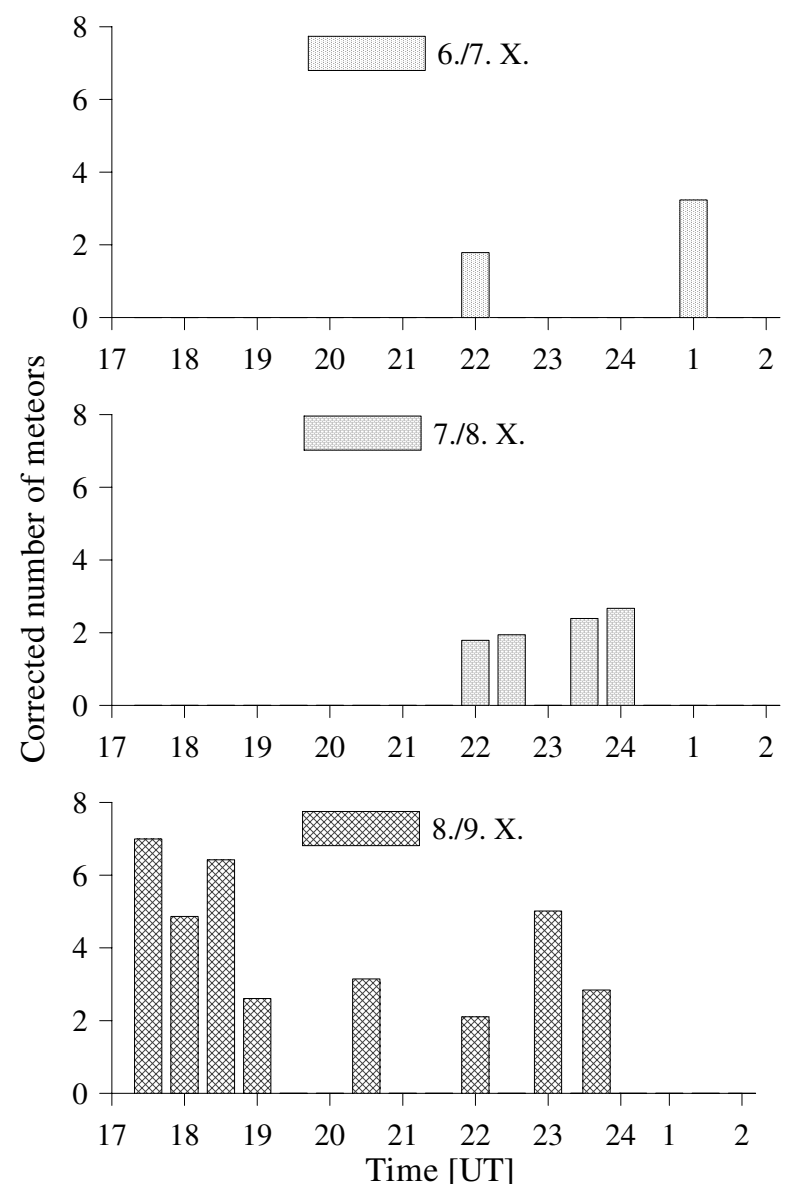

Fig. 1. Activity of the Draconid meteors as observed from the Ondřejov station using a narrow-field camera. The plot shows numbers of the Draconids recorded during three consecutive nights in half hour intervals. The bars represent numbers corrected for the zenith distance of the radiant as well as for the cloudiness.

distribution index of the Draconid meteors $s=1.78 \pm 0.15$. Using only meteors contributing to the peak of the activity, we received a somewhat higher value of $s=1.87 \pm 0.15$.

Our value is not in contradiction with Campbell-Brown et al. (2006), who reported $s=2.0 \pm 0.1$ for the 2005 outburst. Watanabe et al. (1999) derived $s=1.81 \pm 0.36$ for the 1998 Draconid outburst using the HDTV technique. Šimek \& Pecina (1999) reported even lower s values for the 1998 maximum observed by radar. The 1985 return was reportedly richer in faint meteors, the $s$ being between 2.0 and 2.1 (Šimek 1986, 1994). Visual observations tend to give higher $s$ values for both 1985 and 1998 returns (Koseki 1990; Arlt 1998), but they may suffer from uncertain detection probability corrections. For the major Draconid storms of 1933 and 1946, visual observations gave $s$ values about 2 (Watson 1934; Kresák \& Slančíková 1975), while the photographic observation in 1946 gave lower $s$ values (Jacchia et al. 1950).

From our limited data we conclude that the mass distribution of the 2005 Draconid meteoroids was not significantly different from the 1998 return. The comparison with the literature is somewhat difficult because most values cited above were derived from either maximum meteor magnitude or radar echo duration, not from individually determined meteoroid masses, as was done in our case.

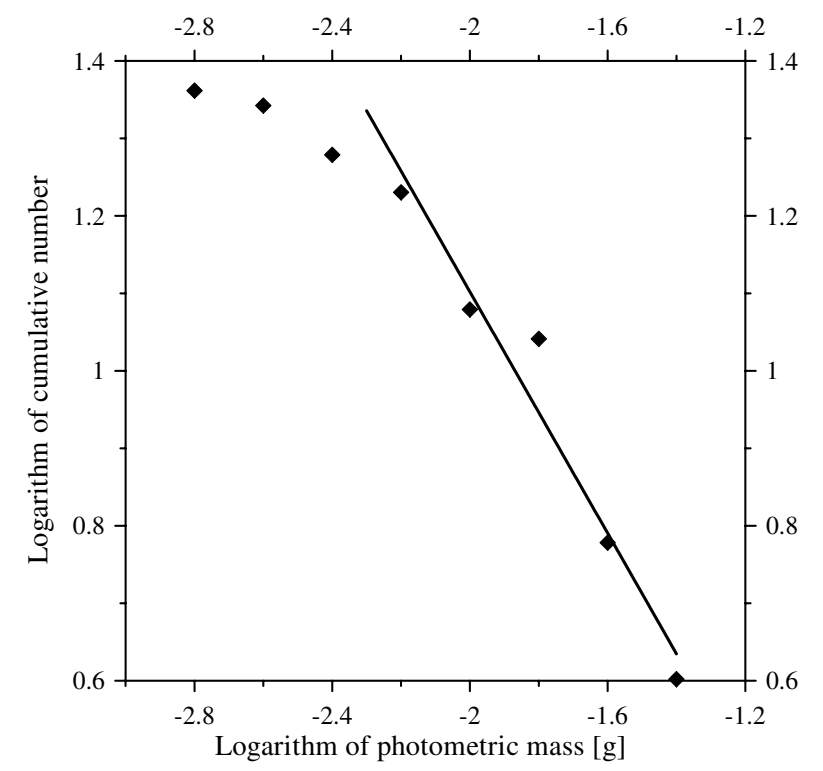

Fig. 2. Mass distribution plot for the Draconid meteors. The faintest meteors are not used for the linear fit.

\subsection{Radiants}

The geocentric radiant distribution of double-station Draconid meteors is presented in Fig. 3. In addition to our video and photographic data, the results of the radar observation and numerical modeling presented in Campbell-Brown et al. (2006) are also included in the plot. We can see that all radiants of optically detected meteors lie within one degree of the theoretical radiant, which was computed for the particles ejected from the parent comet in 1946. The comparison with the results of Campbell-Brown et al. (2006) is given in Table 5. The optical data are in significantly better agreement with the theoretical model than the radar data.

We can only speculate what causes the offset of the radar radiants. It may be an instrumental effect or effect of different meteoroid masses. Note that most of our double station meteors do not actually belong to the outburst, since they were observed after 20 UT. The exceptions are the 05A0830 meteor and the photographic meteor. Both fit well. The cluster of radiants around the theoretical radiant also coincides well with the Draconid radiants observed during the 1998 outburst (Tomita et al. 1999; Suzuki et al. 1999; Fujiwara et al. 2001).

\subsection{Beginning heights}

Figure 4 shows the beginning heights of Draconids in comparison with sporadic meteors of similar velocity as a function of photometric mass. We searched our database and found 55 sporadic meteors with geocentric velocities between 17 and $24 \mathrm{~km} \mathrm{~s}^{-1}$, which are similar to the geocentric velocity of the Draconids. The beginning heights of the Draconid meteors lie between 100 and $110 \mathrm{~km}$. The photographic meteor was not included because of much lower sensitivity of the photographic cameras. In comparison with other meteors with similar geocentric velocity, Draconids are among the highest. The vast majority of sporadic meteors start their luminous trajectories lower than Draconids. If the beginning height reflects the structure of the meteoroids, then the Draconid meteoroids belong to the most fragile material. 


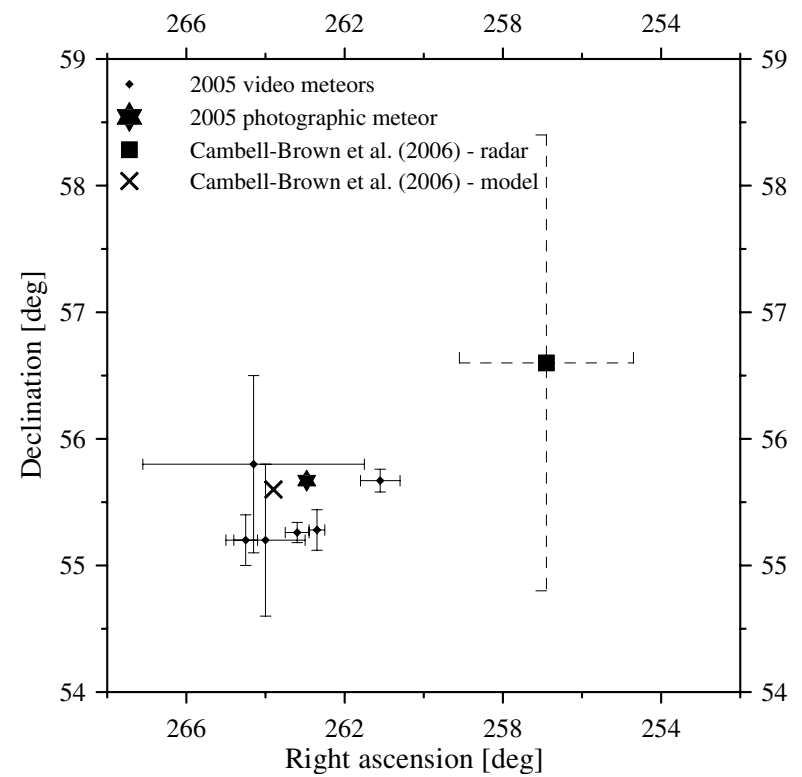

Fig. 3. Radiant distribution of Draconid meteors. Meteors recorded by videocameras are represented by small black diamonds. The mean radiant determined from radar observations by Campbell-Brown et al. (2006) is shown as a black square, whereas theoretical radiant of the modeled stream filament from 1946 (the same paper) is shown as a big cross.

Table 5. Radiants of the Draconid meteors from different sources. (C-B) refers to the paper of Campbell-Brown et al. (2006).

\begin{tabular}{llll}
\hline \hline Source & $\alpha_{\mathrm{G}}$ & $\delta_{\mathrm{G}}$ & $v_{\mathrm{G}}$ \\
& {$\left[{ }^{\circ}\right]$} & $\left.{ }^{\circ}\right]$ & {$\left[\mathrm{km} \mathrm{s}^{-1}\right]$} \\
\hline mean of 6 video meteors & 263.3 & +55.4 & 21.0 \\
photographic meteor & 262.96 & +55.67 & 20.93 \\
radar meteors (C-B) & 256.9 & +56.6 & 19.9 \\
1946 filament model (C-B) & 263.8 & +55.6 & 20.86 \\
\hline
\end{tabular}

Meteor showers of cometary origin usually exhibit increasing beginning height with increasing photometric mass (Koten et al. 2004). Figure 4 indicates that such an increase may also occur in the case of Draconid meteors. Nevertheless, there are only six double station Draconid meteors used for this kind of plot, thus we cannot confirm this hint at the moment. Because of the uncertainty of the single station data, we avoid using these data for the decision. The data of Fujiwara et al. (2001) seem to confirm the height increase.

The beginning height of the meteor can be affected by the different zenith distance of the radiant as well as the different initial velocity. To avoid this influence, we used the parameter $K_{\mathrm{B}}$, which eliminates the potential effect of both quantities. This parameter transforms the problem of classification into a onedimensional parameter as defined by the equation

$K_{\mathrm{B}}=\log \rho_{\mathrm{B}}+2.5 \log v_{\infty}-0.5 \log \cos z_{\mathrm{R}}+0.15$,

where $\rho_{\mathrm{B}}$ is the atmospheric density $\left[\mathrm{g} \mathrm{cm}^{-3}\right]$ at the beginning height $H_{\mathrm{B}}, v_{\infty}$ is the initial velocity $\left[\mathrm{cm} \mathrm{s}^{-1}\right]$, and $z_{\mathrm{R}}$ is the zenith distance of the radiant (Ceplecha 1988). Lower $K_{\mathrm{B}}$ means more fragile meteoroids.

According to Ceplecha (1988) meteoroids with $K_{\mathrm{B}}<6.6$ belong to group D formed by the "soft cometary material". The exact boundary value of $K_{\mathrm{B}}$ may, however, slightly depend on the observational technique. Draconids have been used as a prototype of group D. Figure 5 shows the comparison of Perseid,

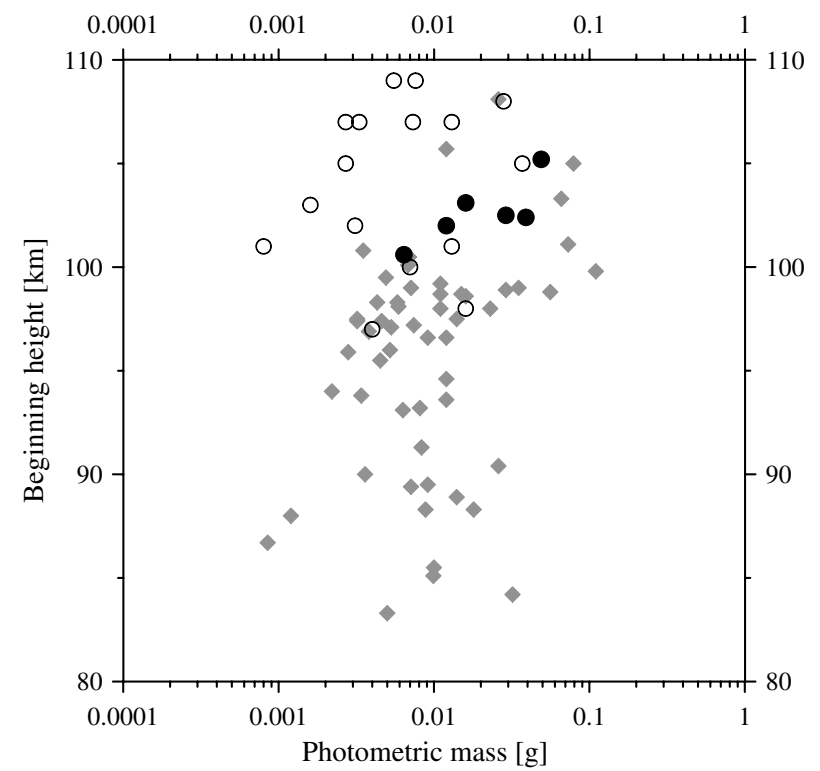

Fig. 4. Beginning heights of Draconid meteors compared with sporadic meteors of similar geocentric velocity. Draconids are shown as circles (double station meteors as full circles and single station ones as open circles), whereas grey diamonds represent the sporadic meteors.

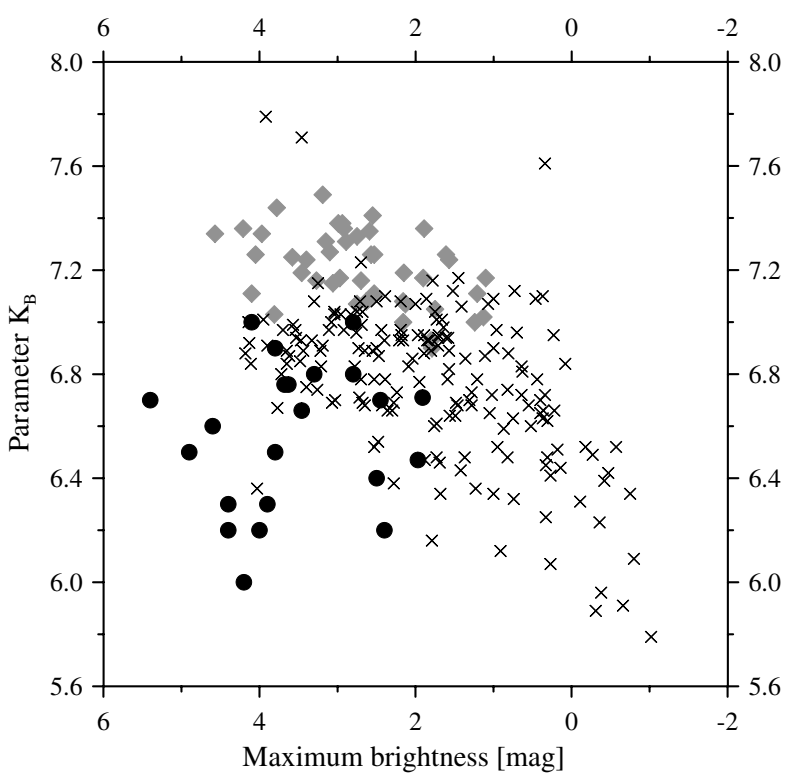

Fig. 5. Parameter $K_{\mathrm{B}}$ of Draconid meteors (black circles) compared with Perseid (crosses) and Quadrantid (grey diamonds) meteors.

Quadrantid, and Draconid meteors. Among meteors with similar maximum brightness, Draconids belong to the group of meteors with the smallest value of $K_{\mathrm{B}}$. There are also Perseid meteors in this figure with comparable values of $K_{\mathrm{B}}$, but these are brighter meteors with higher beginning heights that influence the values of $K_{\mathrm{B}}$.

\subsection{Light curves}

Another characterization of a meteor can be determined from its light curve. If the meteor reaches the maximum brightness later in its luminous trajectory, it is considered that meteoroid was a rather compact body. On the other hand, dust ball meteoroids should produce light curves, which are nearly 
Table 6. Comparison of mean values of the parameter $F$ for different meteor showers.

\begin{tabular}{llll}
\hline \hline $\begin{array}{l}\text { Meteor } \\
\text { shower }\end{array}$ & $\begin{array}{l}\text { Number } \\
\text { of meteors }\end{array}$ & $\begin{array}{l}\text { Parameter } \\
F\end{array}$ & $\begin{array}{l}\text { Source } \\
\text { of data }\end{array}$ \\
\hline Draconids & 17 & 0.46 & \\
Leonids 2000 & 72 & 0.49 & $(1)$ \\
Quadrantids & 28 & 0.50 & $(2)$ \\
Taurids & 42 & 0.54 & $(1)$ \\
Perseids & 160 & 0.54 & $(1)$ \\
Orionids & 108 & 0.55 & $(1)$ \\
Geminids & 64 & 0.58 & $(1)$ \\
\hline
\end{tabular}

(1) Koten et al. (2004).

(2) Koten et al. (2006).

symmetrical (Hawkes \& Jones 1975). Previous studies already showed that video meteors indeed exhibit light curves closer to the symmetrical ones (Murray et al. 1999; Campbell et al. 2000; Koten et al. 2004), which is generally considered as proof of the dust ball model.

We used the traditional parameter $F$ (e.g., Flemming et al. 1993) for the classification of the light curves. The parameter expresses the position of the maximum along the light curve. In addition to the double station meteors, for which the calculation of the absolute meteor brightness was straightforward because of known atmospheric trajectory, we included some of the single station meteors, too. Using their estimated heights and distances, we constructed the light curve in the absolute brightness scale. This procedure significantly increased the number of available meteor light curves to 17 . Of course, only complete light curves can be characterized in this way. The photographic meteor was excluded from this analysis because of its much larger mass and different atmospheric behavior.

Light curves of the Draconid meteors exhibit wide scatter of the $F$ values between 0.24 and 0.70 . We observed also similar scatter among other meteor showers (Koten et al. 2004). Generally, we can say that the majority of the light curves are symmetrical with parameter $F$ closing to the value of 0.5 . The mean value for all 17 meteors is $\bar{F}=0.46 \pm 0.10$. This value is the lowest among all meteor showers characterized this way (Table 6). Again, the Draconid meteors are produced by particles, which belong to the most fragile among all meteor streams.

Figure 6 provides a more detailed look at the distribution of the parameter $F$. Besides the Draconid meteors, Leonids and Geminids are also shown. Although the histograms of all meteor showers reach the maximum in the interval between 0.5 and 0.6 , there are substantial differences among all three distributions. The Draconids show an excess of meteors with $F<0.5$. For higher $F$, the number of meteors steeply decreases and there are no Draconid meteors with $F>0.7$. Thus the histogram is not as symmetrical as is usual among other meteor showers. The Geminids show an opposite pattern with a lack of meteors below $F=0.3$ and many meteors with $F>0.5$. The Leonids lie between these two showers.

\section{Discussion}

On the basis of the video observation of the Draconids meteor shower in 2005 we can confirm the enhanced activity reported by Campbell-Brown et al. (2006). The number of optically detected meteors was not as high as the radar (i.e., fainter) meteors. Apparently, we were able to observe only the descending branch of the reported peak. Despite unfavorable weather

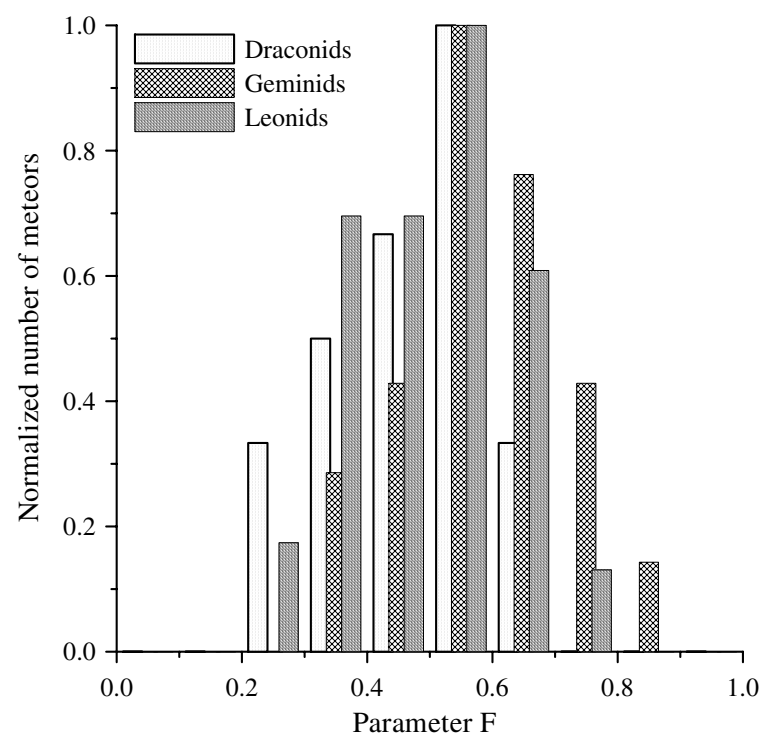

Fig. 6. Histograms of the parameter $F$ for the Draconid (faintly dotted bars), Geminid (crossed bars), and Leonid (black dotted bars) meteors.

conditions it is clear that the enhanced activity ceased around 19 UT on October 8. Later the hourly numbers of meteors did not exceed annual Draconid counts. Scattered Draconid meteors were also observed during the two previous nights. The determination of the mass distribution index suffered from low number statistics, nevertheless, it seems that the index was similar to that in 1998 and somewhat lower than that in 1985.

The radiants of several Draconids recorded simultaneously from two stations lie close to the theoretical value of Campbell-Brown et al. (2006) and also to the radiants reported in other papers describing earlier Draconid outbursts. We did not observe meteors from the radiant detected by the radar, which was shifted by about 4 degrees to the West. Unusual properties of Draconids were already recognized by Jacchia et al. (1950) on the basis of more than 200 photographic meteors recorded in 1946. They found that the beginning heights of the Draconid meteors are significantly higher in comparison with other meteors of comparable geocentric velocity. Moreover the Draconids were also characterized by exceptionally short trails according to their paper. Jacchia et al. (1950) concluded that the meteoroids observed in 1946 were composed of softer than usual material. A similar conclusion was also reached by other authors. Beech (1986) analyzed photographic data from several other papers and found good agreement with the dust ball model of Hawkes \& Jones (1975). According to his paper, the energy necessary for the fragmentation of the unit mass is significantly lower than a similar value found for other meteor showers.

Our video results are in very good agreement with all previous photographic observations of the Draconid meteors. It is necessary to note that video cameras cover lower photometric masses (i.e., fainter meteors) than photographic ones. Nevertheless we found higher than usual beginning heights, too. Also, the values of the parameter $K_{\mathrm{B}}$ are lower in comparison with the same quantity of other meteor showers. Finally, faint Draconid meteors reach the maximum brightness earlier in their luminous trajectory than showers such as Perseid, Orionid, or Leonids do. Thus, all these features confirm the fragile nature of the Draconid meteoroids. The structure of Draconid meteoroids will be further discussed in the forthcomming paper (Borovička et al. 2007). 
Acknowledgements. This work was supported by the Grant Agency of the Academy of Sciences of the Czech Republic grant number B300030502, by the Grant Agency of the Czech Republic grant number 205/05/0543, and by the Academy of Sciences of the Czech Republic scientific project AV0Z10030501. Authors would like to acknowledge the helpful comments of the referee, Dr. P. B. Babadzhanov.

\section{References}

Arlt, R. 1998, JIMO, 26, 256

Beech, M. 1986, AJ, 91, 159

Borovička, J., Spurný, P., \& Koten, P. 2007, A\&A, in preparation

Campbell, M. D., Brown, P. G., LeBlanc, A. G., et al. 2000, M\&PS, 35, 1259

Campbell-Brown, M. D., Vaubaillon, J., Brown, P., Weryk, R., \& Arlt, R. 2006, A\&A, 451, 339

Ceplecha, Z. 1988, Bull. astr. Inst. Czechosl., 39, 4, 221

Davies, J. G., \& Lovell, A. C. B. 1955, MNRAS, 343, 775

Hawkes, R. L., \& Jones, J. 1975, MNRAS, 173, 339

Fleming, F. E. B., Hawkes, R. L., \& Jones, J. 1993, in Meteoroids and their parent bodies, ed. J. Štohl, \& I. P. Williams, Bratislava, 261

Fujiwara, Y., Ueda, M., Sugimoto, M., et al. 2001, in Proc. of the Meteoroids 2001 Conf., ed. B. Warmbein, ESA SP-495, Noordwijk, 123
Jacchia, L. G., Kopal, Z., \& Millman, P. M. 1950, ApJ, 111, 104

Jenniskens, P. 1995, A\&A, 295, 206

Koseki, M. 1990, Icarus, 88, 122

Koten, P. 2002, in Proc. of the Asteroids, Comets, Meteors 2002 Conf., ed. B. Warmbein, ESA SP 500, Berlin, 197

Koten, P., Borovička, J., Spurný, P., Betlem, H., \& Evans, S. 2004, A\&A, 428, 683

Koten, P., Borovička, J., Spurný, P., et al. 2006, MNRAS, 366, 1367

Kresák, L., \& Slančíková, J. 1975, Bull. astr. Inst. Czechosl., 26, 327

Molau, S. 1999, in Proc. of the Meteoroids 1998 Conf., ed. W. J. Baggalay, \& V. Porubčan, Bratislava, 131

Murray, I. S., Hawkes, R. L., \& Jenniskens, P. 1999, M\&PS, 34, 949

Southworth, R. B., \& Hawkins, G. S. 1963, Smithson. Contr. Astrophys., 7, 261

Šimek, M. 1986, Bull. astr. Inst. Czechosl., 37, 246

Śimek, M. 1994, A\&A, 284, 276

Šimek, M., \& Pecina, P. 1999, A\&A, 343, L94

Spurný, P., Borovička, J., \& Shrbený, L. 2007, in Near Earth Objects, our Celestial Neighbors: Opportunity and Risk, ed. G. B. Valsecchi, A. Milani, \& D. Vokrouhlický, IAU Symp., 236, in press

Suzuki, S., Akebo, T., Yoshida, T., \& Suzuki, K. 1999, JIMO, 27, 214

Tomita, M., Murasawa, A., Shimoda, C., et al. 1999, JIMO, 27, 118

Watanabe, J., Abe, S., Takanashi, M., et al. 1999, Geophys. Res. Lett., 26, 1117

Watson, F. Jr. 1934, Harvard College Observatory Bulletin, 895, 9 